\title{
Estresse oxidativo e micronutrientes na hanseníase
}

\section{Oxidative stress and micronutrients}

in leprosy

\author{
Fabiana Maciel de OLIVEIRA ${ }^{1}$ \\ Fernando BARBOSA JÚNIOR² \\ Alceu Afonso JORDÃO JÚNIOR ${ }^{3}$ \\ Norma Tiraboschi FOSS 3 \\ Anderson Marliere NAVARRO ${ }^{4}$ \\ Marco Andrey Cipriani FRADE ${ }^{3}$
}

\section{R E S U M O}

\section{Objetivo}

Avaliar o estresse oxidativo, perfil antioxidante e de micronutrientes em pacientes portadores de hanseníase multibacilar e paucibacilar antes do tratamento poliquimioterápico.

\section{Métodos}

Analisaram-se 52 amostras de soro de pacientes portadores de hanseníase - 38 multibacilares e 14 paucibacilares -, usuários do ambulatório de dermatologia de um hospital público universitário, além de 30 amostras controles. Quantificaram-se marcador de peroxidação lipídica malondialdeído pelo método de substâncias reativas ao ácido tiobarbitúrico, antioxidante glutationa reduzida pelo método baseado na quantificação de tiol solúvel em ácido, antioxidante vitamina E por cromatografia líquida de alta eficiência, minerais selênio, zinco, cobre, magnésio por espectrometria de massa com fonte plasma acoplado, e sorologia do anticorpo glicolipídio fenólico I pelo método Enzyme-Linked Immunosorbent Assay. Foi utilizado teste não paramétrico de Mann-Whitney para comparar as variáveis quantificadas neste estudo entre os diferentes grupos, e correlação de Pearson para verificar associação dessas variáveis com o anticorpo. O critério de significância adotado foi de $p<0,05$.

\footnotetext{
${ }^{1}$ Centro Universitário Católico Salesiano Auxilium, Curso de Nutrição. Araçatuba, SP, Brasil.

2 Universidade de São Paulo, Faculdade de Ciências Farmacêuticas, Departamento de Toxicologia e Essencialidade de Metais. Ribeirão Preto, SP, Brasil.

3 Universidade de São Paulo, Faculdade de Medicina, Departamento de Clínica Médica. Ribeirão Preto, SP, Brasil.

${ }^{4}$ Universidade Estadual Paulista Júlio de Mesquita Filho, Faculdade de Ciências Farmacêuticas, Departamento de Alimentos e Nutrição. Rod. Araraquara-Jaú, Km 1, 14801-902, Araraquara, SP, Brasil. Correspondência para/Correspondence to: AM NAVARRO.E-mails: <navarro@fmrp.usp.br>; <fabi-nut@hotmail.com>.

Artigo baseado na dissertação de FM OLIVEIRA, intitulada "Perfil sérico de estresse oxidativo, antioxidantes e micronutrientes em pacientes com hanseníase". Universidade Estadual Paulista Júlio de Mesquita Filho; 2012.

Apoio: Fundação de Amparo à Pesquisa do Estado de São Paulo (Bolsa auxilio no 2010/14535-5 e no 2011/11704-3).
} 


\section{Resultados}

Houve diferença significativa para o malondialdeído $(p<0,001)$ e vitamina $E(p<0,001)$ no grupo controle comparado aos grupos com hanseníase, multibacilar e paucibacilar. No entanto, essas mesmas variáveis não diferiram entre os grupos multibacilar e paucibacilar ( $p=0,495$ e $p=0,920$ respectivamente). A glutationa reduzida foi superior no grupo controle em relação ao grupo com hanseníase $(p=0,012)$ e multibacilar $(p=0,001)$, no entanto não diferiu do grupo paucibacilar $(p=0,920)$. Quando comparada com os multibacilares e paucibacilares, a glutationa reduzida também não diferiu $(p=0,063)$. Quanto aos minerais, todos se apresentaram dentro da normalidade, exceto o magnésio, cujos níveis foram deficientes em todos os pacientes do estudo. Não foi possível observar correlação do anticorpo glicolipídio fenólico I com as demais variáveis.

\section{Conclusão}

Os pacientes paucibacilares parecem possuir maior defesa antioxidante de glutationa reduzida, semelhante à de indivíduos saudáveis. Baixos níveis de vitamina E nos pacientes com hanseníase são sugestivos dos benefícios de suplementação. As alterações metabólicas observadas não evidenciaram relação com a sorologia do glicolipídio fenólico I.

Palavras-chave: Antioxidantes. Estresse oxidativo. Hanseníase. Micronutrientes.

\section{A B S T R A C T}

\section{Objective}

To determine the oxidative stress and the antioxidant and micronutrient profile of patients with multibacillary and paucibacillary leprosy before polychemotherapeutic treatment.

\section{Methods}

Thirty control samples and fifty-two serum samples from leprosy patients who attended the dermatology outpatient clinic of a public university hospital were analyzed; 38 of them had multibacillary and 14 paucibacillarty. Malondialdehyde, a marker of lipid peroxidation, was determined using the thiobarbituric acid reacting substances assay; the antioxidant reduced glutathione was determined using a method based on the quantification of acid-soluble thiol; the antioxidant vitamin E was determined using High-Performance Liquid Chromatography; the minerals selenium, zinc, copper and magnesium were determined using coupledmass spectrometry, and the serum phenol I glycolipid antibody was determined using Enzyme-Linked Immunosorbent Assay. The nonparametric Mann-Whitney test was used to compare the variables quantified in the present study between the different groups, and Pearson's correlation analysis was used to verify the association between these variables and the antibody. The significance level was set at $p<0.05$.

\section{Results}

There was a significant difference in the content of malondialdehyde $(p<0.001)$ and vitamin $E(p<0.001)$ between the groups with multibacillary and paucibacillary leprosy ( $p=0.495$ and $p=0.920$, respectively) and the control groups. Reduced glutathione levels were higher in the control group compared with those of the group with leprosy $(p=0.012)$ and multibacillary leprosy $(p=0.001)$, but did it not differ from that of the paucibacillary group $(p=0.920)$. Reduced glutathione levels did not differ between the multibacillary and paucibacillary groups ( $p=0.063$ ) either. All minerals were within normal limits, except for magnesium; magnesium deficiency was detected in all groups studied. No correlation was observed between the anti-phenolic glycolipid I antibody and the remaining variables.

\section{Conclusion}

Paucibacillary patients seem to have more pronounced reduced glutathione antioxidant defense, similar to that of healthy individuals. The low vitamin E levels of leprosy patients suggest the benefits of supplementation. The metabolic changes observed did not demonstrate an association with anti-phenolic glycolipid I serology.

Keywords: Antioxidants. Oxidative stress. Leprosy. Micronutrients.

\section{N T R O D U Ç Ã O}

A hanseníase é uma doença infectocontagiosa, de evolução crônica, cujo agente etioló- gico é o bacilo Mycobacterium leprae ( $M$. leprae), microrganismo com tropismo para os nervos periféricos, de alta infectividade e baixa patogenicidade ${ }^{1}$. 
Diante do amplo espectro de manifestações clínicas e imunopatológicas, a hanseníase recebe diversas e complexas classificações. Nesse sentido, em 1982, a World Health Organization (WHO) propôs uma classificação a fim de expandir a campanha de eliminação da doença, sendo utilizada até hoje devido ao seu caráter simplificado e operacional. Essa classificação se baseia na contagem do número de lesões de pele e nervos envolvidos: agrupam-se em paucibacilares quando apresentam de uma a cinco lesões e incluem as formas clínicas indeterminada e tuberculoide localizadas no polo tuberculoide ou em multibacilares quando apresentam mais de cinco lesões e incluem as formas clínicas dimorfa tuberculoide, dimorfa dimorfa, dimorfa virchowiana e virchowiana localizadas no polo virchowiano ${ }^{1,2}$.

O diagnóstico tardio e/ou manejo terapêutico inadequado podem levar ao surgimento de sequelas ou incapacidade permanente, o que traz sérias consequências para o sistema previdenciário, uma vez que a doença atinge em sua maioria a faixa etária economicamente ativa da população. Por isso, a constante vigilância para novos casos, em especial nos menores de 15 anos (utilizado como indicador de endemia), e acompanhamento daqueles já existentes são imprescindíveis ${ }^{3,4}$.

Embora estabelecidos a cura e o tratamento para a doença, disponível gratuitamente à população, a hanseníase ainda permanece como um grave problema de saúde pública. Segundo dados recentes, a prevalência da doença no Brasil é de 1,51 casos/10 mil habitantes, e o país ocupa o segundo lugar no número de casos no mundo, atrás somente da Índia. Vale ressaltar que, apesar da redução de alguns indicadores observada nos últimos anos, o padrão espacial da doença permanece o mesmo, ou seja, ainda persistem regiões hiperendêmicas associadas à pobreza e a baixos índices de desenvolvimento humano ${ }^{5,6}$.

O uso do Anti-Phenolic Glycolipid I Sorology (APGLI, Sorologia do Glicolipídio Fenólico I), que é um antígeno específico da parede do $M$. leprae e prediz a carga bacilar, tem sido útil para iden- tificar indivíduos de maior risco e indica infecção do tipo multibacilar 6 . Além disso, a literatura demostra aumento de estresse oxidativo, depleção antioxidante e de micronutrientes na hanseníase ${ }^{7-11}$. Contudo, os mecanismos que explicam tais alterações permanecem obscuros, e associá-los à causa ou consequência da doença pode contribuir de forma significativa para o diagnóstico preciso e precoce, tratamento, reabilitação e manejo da doença como um todo.

O presente estudo teve por objetivo avaliar o estresse oxidativo, antioxidantes, micronutrientes e APGLI em pacientes portadores de hanseníase a fim de elucidar possíveis mecanismos associados à suscetibilidade e à patogenia da doença.

\section{M É T O D O S}

Trata-se de um estudo transversal, cujo Protocolo foi aprovado pelo Comitê de Ética e Pesquisa do Hospital das Clínicas e da Faculdade de Medicina de Ribeirão Preto, Universidade de São Paulo (Of. Ref. n 4365/2011).

A amostra foi constituída por 52 pacientes portadores de hanseníase multibacilares e paucibacilares, usuários do ambulatório de dermatologia de um hospital público universitário. Os critérios de inclusão foram idade adulta ( $\geq 19$ e $<60$ anos), diagnóstico confirmado para hanseníase, ausência de comorbidade e não estar em uso do tratamento poliquimioterápico (casos novos ou de recidiva). Em virtude da não existência de valores de referência para marcadores de estresse oxidativo, foi selecionado um grupo controle composto por 30 indivíduos, com os seguintes critérios de inclusão: idade adulta, saudáveis e/ou ausência de comorbidades.

Foram dosados sorologicamente o marcador de peroxidação lipídica malondialdeído, antioxidantes Glutationa Reduzida (GSH) e vitamina E nos pacientes com hanseníase e indivíduos do grupo controle. A sorologia do APGLI e selênio (Se), zinco (Zn), cobre (Cu) e magnésio (Mg) foram 
dosados nos pacientes com hanseníase, sendo os minerais comparados segundo valores de referências da literatura.

O malondialdeído foi quantificado pelo método Thiobarbituric Acid Reactive Substances (TBARS, Substâncias Reativas ao Ácido Tiobarbitíruco) ou teste de substâncias reativas ao ácido tiobarbitúrico ${ }^{12}$; a GSH foi quantificada pelo método baseado na quantificação de tiol solúvel em ácido que é lido em espectrofotômetro no comprimento de onda de $640 \mathrm{~nm}^{13}$, e a vitamina $\mathrm{E}$ foi dosada por meio da cromatografia líquida de alta eficiência em coluna tipo C-18 (Shimpack CLC-ODS $4,6 \times 25 \mathrm{~cm}$ ), pré-coluna $4 \mathrm{~mm} \times 1 \mathrm{~cm}$ e fluxo de $2,0 \mathrm{~mL} / \mathrm{min}^{14}$. A sorologia do APGLI foi dosada por ensaio imunoenzimático, Enzyme-Linked Immunosorbent Assay (ELISA) ${ }^{15}$.

Os minerais Se, Zn, Cu e Mg foram dosados por meio da espectrometria de massa com fonte de plasma acoplado ${ }^{16}$ e avaliados segundo valores de referência da literatura, sendo 0,44-1,16 $\mu \mathrm{mol} / \mathrm{L}$ para o Se; 10,4-22,8 $\mu \mathrm{mol} / \mathrm{L}$ para o Zn; 7,9-25,1 $\mu \mathrm{mol} / \mathrm{L}$ para o $\mathrm{Cu}^{17}$ e 800-1200 $\mu \mathrm{mol} / \mathrm{L}$ para o $\mathrm{Mg}^{18}$.

Foi aplicado o teste não-paramétrico Mann-Whitney para comparar as variáveis malondialdeídos, GSH e vitamina E entre os grupos controle versus hanseníase, multibacilares e paucibacilares, e entre os doentes multibacilares versus paucibacilares. Teste de correlação Pearson foi realizado a fim de verificar associação do APGLI com as demais variáveis quantificadas (malondialdeído, GSH, vitamina E, Se, Zn, Cu e Mg). As análises foram realizadas em programa estatístico que utilizou p<0,05 como critério de significância.

\section{RESULTADOS}

Do total de 52 pacientes com hanseníase incluídos no estudo, 73,0\% ( $n=38)$ eram multibacilares e $27,0 \%(n=14)$ paucibacilares, sendo $74,2 \%$ ( $n=36)$ homens e $25,8 \%(n=16)$ mulheres, com média de idade igual a 45,2 $\pm 9,7$ anos (21-59). O grupo-controle $(n=30)$ apresentou média de idade igual a $24,7 \pm 4,3$ anos (20-43), sendo $43,3 \%$ $(n=13)$ homens e $56,7 \%(n=17)$ mulheres.

Em relação à sorologia do APGLI, o grupo controle apresentou valor médio $(0,5 \pm 0,2)$ inferior $(p<0,001)$ ao grupo com hanseníase $(5,6 \pm 6,2)$, e dentre os doentes, o grupo multibacilares apresentou valor médio $(7,4 \pm 6,4)$ superior $(p<0,001)$ ao grupo paucibacilares $(1,0 \pm 0,6)$.

Os resultados de mediana, valor mínimo e máximo do malondialdeído, GSH e vitamina $\mathrm{E}$ quantificados nos grupos com hanseníase e controle estão descritos na Tabela 1. As diferenças de tamanho amostral " $n$ " ocorreram devido ao esgotamento não previsto de material biológico (soro) durante as análises em laboratório.

Tabela 1. Número amostral (n), Mediana (Md) e valores mínimo e máximo (min-max) do Thiobarbituric Acid Reactive Substances (TBARS), Glutationa Reduzida (GSH) e vitamina E referentes aos grupos controle, com hanseníase, multibacilar e paucibacilar. Ribeirão Preto (SP), 2012

\begin{tabular}{|c|c|c|c|c|c|c|}
\hline \multirow{2}{*}{ Grupos } & \multicolumn{6}{|c|}{ Valores em $\mu \mathrm{mol} / \mathrm{L}$ : Md; min-max } \\
\hline & $\mathrm{n}$ & TBARS & $\mathrm{N}$ & GSH & $\mathrm{n}$ & Vitamina $\mathrm{E}$ \\
\hline Controle & 30 & 5,$9 ; 2,0-13,5$ & 30 & 374,$5 ; 214,5-807,0$ & 30 & 13,$9 ; 8,5-24,8$ \\
\hline Hanseníase & 40 & 13,$8 ; 5,9-33,3$ & 52 & 318,$9 ; 145,9-629,5$ & 38 & 3,$9 ; 1,1-11,3$ \\
\hline Valor de $p^{*}$ & & $<0,001$ & & 0,012 & & $<0,001$ \\
\hline Multibacilar & 26 & 13,$5 ; 5,9-22,8$ & 38 & 312,$1 ; 145,9-564,5$ & 32 & 3,$9 ; 1,11-11,3$ \\
\hline Valor de $p^{*}$ & & $<0,001$ & & 0,001 & & $<0,001$ \\
\hline Paucibacilar & 14 & 14,$7 ; 9,2-33,3$ & 14 & 392,$0 ; 174,5-629,5$ & 6 & 4,$6 ; 1,3-7,0$ \\
\hline Valor de $p^{*}$ & & $<0,001$ & & 0,977 & & $<0,001$ \\
\hline Valor de $p^{* *}$ & & 0,495 & & 0,063 & & 0,920 \\
\hline
\end{tabular}

Nota: ${ }^{*}$ Comparação do grupo controle versus grupos com hanseníase, multibacilar e paucibacilar; ${ }^{* *}$ Do total de doentes (grupo com hanseníase), comparação do grupo multibacilar versus grupo paucibacilar. 
O malondialdeído foi inferior $(p<0,001)$ no grupo controle quando comparado ao grupo com hanseníase, multibacilares e paucibacilares. Da mesma forma, a vitamina $\mathrm{E}$ foi superior $(p<0,001)$ no grupo controle quando comparada a esses grupos.

A glutationa reduzida foi estatisticamente superior no grupo controle quando comparada aos grupos com hanseníase $(p=0,012)$ e multibacilares $(p=0,001)$, no entanto, quando comparada ao grupo paucibacilares, não houve diferença significante $(p=0,920)$. Dentre os doentes, não foi possível observar diferenças significantes entre o grupo multibacilares e paucibacilares para as variáveis malondialdeído, GSH e vitamina $\mathrm{E}$.

Na Tabela 2, encontram-se descritos os resultados de média e desvio-padrão dos minerais $\mathrm{Se}, \mathrm{Zn}, \mathrm{Cu}$ e Mg, quantificados no grupo com hanseníase, e a classificação dos pacientes de acordo com valor sérico de referência. O tamanho amostral foi reduzido para um " $n$ " de 28 devido à falta de material biológico não prevista.

Os pacientes com hanseníase apresentaram níveis séricos normais de Se, Zn e Cu, exceto por uma minoria de multibacilares $(n=4$ ou 14,3\%) que apresentou alteração bioquímica de Cu acima da referência. Entretanto, em relação ao $\mathrm{Mg}$, todos os pacientes apresentam-se alterados, com um valor médio de 531,9 $971,2 \mu \mathrm{mol} / \mathrm{L}$, bem abaixo do limite inferior de referência (800-1200 $\mu \mathrm{mol} / \mathrm{L}$ ).

Não foi possível observar correlação significativa da sorologia do APGLI com as variáveis de estresse oxidativo, antioxidantes e minerais quantificadas neste estudo.

\section{I S C U S S Ã O}

O malondialdeído, produto final estável do processo de peroxidação lipídica, é considerado um importante mediador de danos nos tecidos e encontra-se aumentado na hanseníase ${ }^{7-9}$, fenômeno esse corroborado no presente estudo, que demonstrou aumento significativo dos níveis séricos de malondialdeído nos doentes em comparação ao grupo controle, mas que não detectou diferenças entre os grupos multibacilares e paucibacilares.

Da mesma forma, a depleção da defesa antioxidante também foi constatada nesse e em outros estudos que mostraram níveis reduzidos de enzimas e moléculas antioxidantes na hanseníase $^{8,10,11}$.

Prasad et al. ${ }^{11}$ verificaram níveis reduzidos de GSH no sangue de pacientes com a doença em relação ao grupo controle, exceto para as formas tuberculoide e dimorfa tuberculoide, ou seja, formas paucibacilares. No presente estudo, o resultado foi semelhante, uma vez que o grupo paucibacilares apresentou GSH estaticamente igual ao de indivíduos saudáveis, enquanto, no grupo multibacilares, a GSH foi deficiente e poderia estar associada ao perfil de suscetibilidade dos multibacilares diante da hanseníase (baixa resistência e alta disseminação dos bacilos). Sabe-se que a GSH é um importante antioxidante que atua em conjunto com as enzimas glutationa peroxidase e redutase para remoção do peróxido de hidrogênio ${ }^{19}$.

A vitamina $E$, comumente representada pelo $\alpha$-tocoferol, tem sido considerada um impor-

Tabela 2. Média e Desvio-Padrão (DP) do selênio, zinco, cobre e magnésio e número (\%) de pacientes classificados em alterados e não alterados segundo valor sérico de referência de cada mineral. Ribeirão Preto (SP), 2012

\begin{tabular}{lcccc}
\hline \multirow{2}{*}{$\begin{array}{l}\text { Grupo com hanseníase } \\
\mathrm{n}=28\end{array}$} & \multicolumn{4}{c}{ Valor sérico de referência dos minerais em $\mu \mathrm{mol} / \mathrm{L}$} \\
\cline { 2 - 5 } & VR: $0,44-1,16$ & Zinco & Cobre & Magnésio \\
VR: $10,4-22,8$ & $15,65 \pm 2,0$ & $20,6 \pm 3.6$ & $531,9 \pm 71,2$ \\
Média \pm DP $(\mu \mathrm{mol} / \mathrm{L})$ & $0,73 \pm 0,17$ & - & $4,0(14,3)$ & $28,0(100,0)$ \\
Alterado $\mathrm{n}(\%)$ & - & $28,0(100,0)$ & $24,0(85,7)$ & - \\
Não alterado $\mathrm{n}(\%)$ & $28,0(100,0)$ & & & VR: $800,0-1200,0$ \\
\hline
\end{tabular}

Nota: Valores de Referência (VR) de cada mineral foram utilizados como pontos de corte para classificação em alterados e não alterados. 
tante antioxidante protetor da bicamada lipídica das membranas celulares e das lipoproteínas de transporte ${ }^{20}$. Segundo Girish ${ }^{21}$, além de antioxidante, a vitamina $\mathrm{E}$ também exerce um papel protetor no sistema imunológico, o que poderia ser uma boa medida de referência para avaliação do risco de infecção inicial. Redução dos níveis séricos dessa vitamina foi observada nos pacientes com hanseníase neste estudo, em comparação ao grupo controle, no entanto sem diferença significativa entre os grupos da doença. Esses resultados corroboram a literatura, que relata redução dos níveis séricos de vitamina E em pacientes sem tratamento ${ }^{22}$ e em uso da poloquimioterapia por seis meses a um ano ${ }^{21}$.

Vale destacar que a poloquimioterapia se baseia na combinação de três drogas (dapsona, rifampicina e clofazimina), sendo, em 1982, preconizada pela WHO como tratamento para a cura da hanseníase, e, em 1995, fornecida gratuitamente à população de países endêmicos, incluindo o Brasil ${ }^{1,2}$.

Diversos estudos relatam redução e/ou alteração de micronutrientes em doenças crônicas, dentre elas a hanseníase ${ }^{23-26}$. O desequilíbrio de micronutrientes pode gerar prejuízos à função celular, fisiológica e imunológica, sendo tais alterações associadas à causa ou à consequência de inúmeras doenças ${ }^{27}$.

Embora antigos, os estudos que avaliaram minerais na hanseníase mostraram evidências de que as alterações de micronutrientes não estão associadas à alimentação, visto que tais alterações seguem um mesmo padrão nas diferentes formas clínicas da doença ${ }^{22,28}$. Os estudos sugerem que essas alterações estejam associadas à carga bacilar, ou seja, quanto maior o número de bacilos (formas clínicas do polo virchowiano), maiores as alterações ${ }^{24,25}$. No entanto, no presente estudo, não foi possível observar associação de micronutrientes com a carga bacilar inferida pelo APGLI, assim como para as outras variáveis de estresse oxidativo e antioxidantes.

Uma das hipóteses que explica a redução de micronutrientes na hanseníase é a de que os bacilos do $M$. leprae sequestrariam minerais das células do hospedeiro para uso no próprio metabolismo, uma vez que já é conhecida a participação cardinal dos minerais na atuação de inúmeras enzimas ${ }^{25,28,29}$. Como consequência da redução dos minerais no hospedeiro, as metaloenzimas com função antioxidante (superóxido dismutase e glutationa peroxidase) seriam afetadas negativamente ${ }^{11}, \mathrm{e}$, ainda, segundo Jain et al. ${ }^{29}$, as alterações de micronutrientes na hanseníase poderiam ser atribuídas à liberação de interleucina-I, produzida durante processo inflamatório, o que causaria aumento de $\mathrm{Cu}$ e redução de $\mathrm{Zn}$ e ferro no hospedeiro.

No presente estudo, todos pacientes com hanseníase apresentaram níveis séricos normais do mineral Se, segundo valor de referência. No entanto, esses resultados diferem de Foster et al. ${ }^{30}$, que analisaram 40 elementos traços em 14 pacientes com hanseníase e 5 indivíduos saudáveis e observaram redução de Se nos doentes. Vale destacar que uma das principais funções do Se está associada ao seu potencial antioxidante, uma vez que ele atua como cofator da glutationa peroxidase, e a atividade dessa enzima decresce na deficiência de Se, enquanto a suplementação restabelece sua atividade normal ${ }^{31}$.

Estudos também relataram deficiência de Zn, que acompanha o caráter espectral da doença, ou seja, redução gradual do polo tuberculoide (alta resistência) ao polo virchowiano (alta suscetibilidade)22,24,26,28. No presente estudo, todos os pacientes apresentaram níveis séricos normais de Zn. No entanto, vale destacar que o nível plasmático desse mineral não é considerado um bom indicador do seu estado nutricional, uma vez que o organismo tenta manter valores normais durante sua deficiência, ao passo que o Zn eritrocitário, por não refletir mudanças recentes, é considerado um indicador de prazo mais longo ${ }^{32}$.

A literatura também relata Cu aumentado em infecções agudas e crônicas, incluindo a hanseníase ${ }^{24}$, e associa essa alteração à redução de Zn, ou seja, a deficiência de Zn (hipozincemia) levaria ao aumento de $\mathrm{Cu}$ (hipercupremia) ${ }^{26}$, uma 
vez que esses minerais são antagonistas, possivelmente por competirem pelo mesmo transportador de membrana da borda em escova no intestino ${ }^{31}$. Além disso, os estudos relatam que o aumento de Cu em pacientes com hanseníase é crescente ao longo do espectro, ou seja, do polo tuberculoide ao polo virchowiano, onde atinge valores máximos ${ }^{24,26,33}$. No entanto, no presente estudo, a maioria dos pacientes com hanseníase apresentou níveis séricos de Cu normais, sendo somente quatro pacientes multibacilares com níveis moderadamente aumentados desse mineral.

Em relação ao Mg, todos os pacientes com hanseníase apresentaram níveis muito abaixo do limite inferior de referência, indicando uma possível deficiência crônica desse micronutriente. Outros estudos também relataram redução dos níveis de Mg em pacientes com hanseníase, contudo a causa dessa alteração permanece desconhecida ${ }^{26,30,34}$. Estudos recentes têm revelado uma forte relação do $\mathrm{Mg}$ com a inflamação e resposta imune não específica (inata) e específica (adquirida) ${ }^{35}$, mostrando que a deficiência de Mg produz uma síndrome inflamatória com leucócitos e ativação de macrófagos, liberação de citocinas e produção excessiva de radicais livres, e ainda que esse estado pró-inflamatório e pró-oxidante é sistêmico e envolve múltiplos tecidos e órgãos ${ }^{36,37}$. Na hanseníase, a deficiência de $\mathrm{Mg}$ poderia contribuir para o estresse oxidativo, depleção antioxidante e consequente danos nos nervos periféricos.

Segundo Foster et al. ${ }^{30}$, há dados consistentes na literatura sobre o envolvimento metabólico e nutricional na etiologia e patogenia da hanseníase. Nesse sentido, o fato do grupo paucibacilares ter apresentado defesa antioxidante de GSH igual ao grupo controle, mesmo com baixos níveis de vitamina $\mathrm{E}$ e $\mathrm{Mg}$, demonstra um importante achado do presente estudo que pode ser mais explorado, como traçar perfis imunológicos, bioquímicos e nutricionais e comparar os diferentes grupos. Além disso, com um número amostral aumentado, é possível observar diferença estatística de GSH entre multibacilares e pauci- bacilares, que no presente estudo apresentou um $p=0,063$.

O uso da terapia nutricional como estratégia de tratamento da doença também é sugestivo no presente estudo, em especial para vitamina E, que foi inferior nos pacientes com hanseníase. A relação entre doença, nutrição e imunidade tem sido investigada extensamente nos dias atuais, e mostra um aumento do catabolismo diante de uma doença, o que leva a um aumento das necessidades nutricionais visando a assegurar eficiência da resposta imune, sendo esta dependente de uma elevada taxa de replicação celular, síntese de compostos proteicos e demais compostos bioativos.

Uma vez que as células do sistema imune são altamente sensíveis aos danos oxidativos, e que esse tipo de estresse metabólico presente na hanseníase promove aumento das necessidades de antioxidantes (enzimas e moléculas), sugere-se que uma terapia nutricional com suplementação ou dieta específica pode ser benéfica. Alguns estudos revelam essa tendência, porém os resultados são conflitantes devido à falta de padronização e rigor metodológico (dose suplementada, no caso vitamina $E$, tipos da doença, administração da poloquimioterapia, entre outros) $)^{38,39}$. Faz-se necessária a realização de ensaio clínico randomizado duplo cego para se obterem recomendações com alto nível de evidência, bem como estudos para elucidação das respostas imunológicas e inflamatórias da doença.

\section{CONCLUSÃO}

Os pacientes paucibacilares parecem possuir maior defesa antioxidante de GSH em relação aos multibacilares, sendo essa defesa semelhante à de indivíduos saudáveis. Baixos níveis de vitamina E nos pacientes com hanseníase são sugestivos de que uma suplementação poderia ser benéfica no decorrer do tratamento. As alterações metabólicas observadas neste estudo não evidenciaram relação com a sorologia APGLI. 
Novos estudos clínicos e observacionais são necessários para o estabelecimento de marcadores associados à resistência e à suscetibilidade do hospedeiro diante da infecção pelo $M$. leprae, visando também à implementação de terapia nutricional específica para a hanseníase.

\section{COLABORADORES}

FM OLIVEIRA, AM NAVARRO e MAC FRADE participaram na elaboração de estratégia experimental, coleta de dados, tabulação, discussão dos resultados e elaboração do artigo. F BARBOSA JÚNIOR participou das análises de minerais, tabulação e elaboração do artigo. AA JORDÃO JÚNIOR participou das análises bioquímicas, tabulação e elaboração do artigo. NT FOSS participou na elaboração de estratégia experimental, coleta de dados e elaboração do artigo.

\section{REFERÊ NCIAS}

1. Crespor MJI, Gonçalves A, Padovani CR. Haseníase: pauci e multibacilares estão sendo diferentes? Rev Med. 2014; 47(1):43-50.

2. World Health Organization. Leprosy today. Washington (DC): WHO; 2015 [cited 2015 Apr 20]. Available from: http://www.who.int/mediacentre/ factsheets/fs101/en/

3. Marinho FD, Nardi SMT, Coutinho GC, Sime MM. Hanseníase em menores de 15 anos: uma revisão bibliográfica. Refacs. 2015; 3(2):95-105.

4. Pires CA, Malcher CMSR, Abreu Júnior JMC, Albuquerque TG, Correa IRS, Darbacher ELR. Leprosy in children under 15 years: The importance of early diagnosis. Rev Paul Pediatr. 2012; 30(2):292-5.

5. Ribeiro GC, Fabri ACOC, Amaral EP, Machado IE, Lana FCF. Estimativa da prevalência oculta da hanseníase na microrregião de Diamantina - Minas Gerais. Rev Eletr Enf. 2014; 16(4):728-35. http:// dx.doi.org/105216/ree.v16i4.22371

6. Cruz RCS, Cunha MGS, Vásquez FG. Prevalência de anticorpo anti PGL-1 em contatos domiciliares de pacientes com hanseníase. Cad Saúde Colet. 2009; 17(1):261-71.

7. Jyothi P, Riyaz N, Nandakumar G, Binitha MP. A study of oxidative stress in paucibacillary and multibacillary leprosy. Indian J Dermatol Venereol Leprol. 2008; 74(1):80.
8. Reddy YN, Murthy SV, Krishna DR, Prabhakar MC. Oxidative stress and anti-oxidant status in leprosy patients. Indian J Lepr. 2003; 75(4):307-16.

9. Bhadwat VR, Borade VB. Increased lipid peroxidation in lepromatous leprosy. Indian J Dermatol Venerol Leprol. 2000; 66(3):121-5.

10. Abdel-Hafez HZ, Mohamed EEM, Abd-Elghany AA. Tissue and blood superoxide dismutase activity and malondialdehyde level in leprosy. J Eur Acad Dermatol Venereol. 2009; 24(6):704-8.

11. Prasad CVB, Kodliwadmath MV, Kodliwadmath GB. Erythrocyte glutathione peroxidase, glutathione reductase activities and blood glutathione content in leprosy. J Infect. 2008; 56(6):469-73.

12. Buege JA, Aust SD. Microsomal lipid peroxidation. Methods Enzymol. 1978; 52(1):302-10.

13. Sedlak J, Lindsay RH. Estimation of total, proteinbound, and nonprotein sulfhydryl groups in tissue with Ellman's reagent. Anal Biochem. 1968; 25(1):192-205.

14. Arnaud J, Fortis I, Blachier S, Kia D, Favier A. Simultaneous determination of retinol, alphatocopherol and beta-carotene in serum by isocratic high-performance liquid chromatography. J Chromatogr. 1991; 572(1-2):103-16.

15. Buhrer-Sekula S, Sarno EN, Oskam L, Koop S, Wichers I, Nery JA, et al. Use of ML dipstick as a tool to classify leprosy patients. Int J Lepr Other Mycobact Dis. 2000; 68(4):456-63.

16. Nunes JA. Desenvolvimento de método para determinação de $\mathrm{Ag}, \mathrm{As}, \mathrm{Cd}, \mathrm{Co}, \mathrm{Mn}, \mathrm{Ni}, \mathrm{Pb}$ e Se em sangue por espectrometria de massas com fonte de plasma acoplado indutivamente (ICP-MS) utilizando diluição das amostras em meio alcalino [doutorado]. Ribeirão Preto: Universidade de São Paulo; 2009.

17. Rukgauer M, Klein J, Kruse-Jarres JD. Refrence values for the trace elements copper, manganese, selenium, and zinc in the serum/plasma of children, adolescents and adults. J Trace Elem Med Biol. 1997; 11(2):92-8

18. Young DS. Implementation of SI units for clinical laboratory data: Style specifications and conversion tables. Ann Intern Med. 1987; 106(1):10-35.

19. Papp LV, Holmgren A, Khanna KK. Selenium and selenoproteins in health and disease. Antioxid Redox Signal. 2010; 12(7):793-5.

20. Barreiros ALBS, David JM, David JP. Estresse oxidativo: relação entre geração de espécies reativas e defesa do organismo. Quím Nova. 2006; 29(1):113-23.

21. Girish $\mathrm{S}$. Role of antioxidant vitamins in immune function in leprosy. IJCP. 2011; 2(8):1-3. 
22. Rao KN, Saha K. Undernutrition and lepromatous leprosy: Serum vitamin $A$ and $E$ levels in leprosy spectrum. Indian J Lep. 1988; 60(1):66-70.

23. Wang GQ, Lin MY. Serum trace element levels in tuberculous pleurisy. Biol Trace Elem Res. 2011; 141(1-3):86-90.

24. George J, Bhatia VN, Balakrishnan S, Ramu G. Serum zinc/copper ratio in subtypes of leprosy and effect of oral zinc therapy on reactional states. Int J Lepr Other Mycobact Dis. 1990; 59(1):20-4.

25. Manthur NK, Sharma M, Mangal HN, Rai SML. Serum zinc levels in subtypes of leprosy. Inter J Lepr. 1984; 52(3):327-30.

26. Rao KN, Gupta JD, Sehgal VN, Chakrabarti AK, Saha $\mathrm{K}$. Trace elements in the sera of leprosy spectrum. Indian J Lepr. 1985; 57(3):556-61.

27. Liu BY, Bu XM, Wang GQ. Trace elements analysis of urine and hair in tuberculous pleurisy. Biol Trace Elem Res. 2011; 143(3):1319-24.

28. Mennen U, Howells C, Wiese AJ. Serum zinc, sodium, calcium, magnesium and potassium levels and standard diet in leprosy patients. Indian J Lepr. 1993; 65(4):415-21.

29. Jain A, Mukherjee A, Chattopadhya D, Saha K. Biometals in skin and sera of leprosy patients and their correlation to trace element contents of $M$. leprae and histological types of the disease: A comparative study with cutaneous tuberculosis. Inter J Lepr Other Mycobact Dis. 1995; 63(2): 249-58.

30. Foster R, Sanchez A, Foulkes J, Cameron LJ. Profile of blood elements in leprosy patients. Indian J Lepr. 1991; 63(1):12-33.

31. Costa NMB. Minerais. In: Costa NMB, Peluzio MCG. Nutrição básica e metabolismo. Viçosa: UFV; 2008.

32. Mafra D, Cozzolino SMF. Importância do zinco na nutrição humana. Rev Nutr. 2004; 17(1):79-87.
http://dx.doi.org/10.1590/S1415-52732004000 100009

33. Sher R, Shulman G, Path MRC, Baily P, Politzer WM. Serum trace elements and vitamin A in leprosy subtypes. Am J Clin Nutr. 1981; 34(9):1918-24.

34. Nigam P, Mukhija RD, Agrawal AK, Sati TR, Kapoor KK. Serum cations (calcium and magnesium) in leprosy. Indian J Lepr. 1985; 57(3):529-33. http:// dx.doi.org/10.1007/s12028-008-9068-9

35. Tam M, Gomez S, Gonzalez-Gross M, Marcos A. Possible roles of magnesium on the immune system. Eur J Clin Nutr. 2003; 57(10):1193-7.

36. Brilla LR 2012. Magnesium influence on stress and immune function in exercise. J Sports Med Doping Stud. 2012; 2(3):1-3. http://dx.doi.org/10.4172/ 2161-0673.1000111

37. Macêdo EMC, Amorim MAF, Silva ACS, Castro CMMB. Efeitos da deficiência de cobre, zinco e magnésio sobre o sistema imune de crianças com desnutrição grave. Rev Paul Pediatr. 2010; 28(3): 329-36. http://dx.doi.org/10.1590/S0103-0582201 0000300012

38. Vijayaraghavan R, Suribabu CS, Sekar B, Oommen PK, Kavithalakshmi SN, Madhusudhanan N, et al. Protective role of vitamin $\mathrm{E}$ on the oxidative stress in Hansen's disease (Leprosy) patients. Eur J Clin Nutr. 2005; 59(10):1121-8.

39. Rimoli LF. Efetividade da vitamina $E$ na redução do estresse oxidativo em hansenianos da forma multibacilar sob tratamento [doutorado]. São José do Rio Preto: Faculdade de Medicina de São José do Rio Preto; 2006. 
